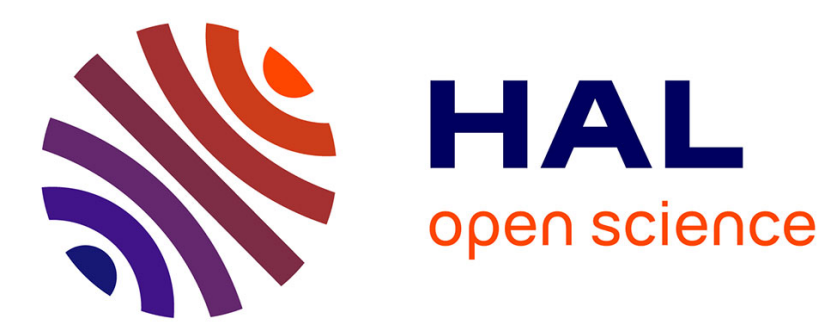

\title{
Perimeter-based coverage optimization to improve lifetime in wireless sensor networks
}

Ali Kadhum Idrees, Karine Deschinkel, Michel Salomon, Raphael Couturier

\section{To cite this version:}

Ali Kadhum Idrees, Karine Deschinkel, Michel Salomon, Raphael Couturier. Perimeter-based coverage optimization to improve lifetime in wireless sensor networks. Engineering Optimization, 2016, 48 (11), pp.1951 - 1972. hal-02131156

\section{HAL Id: hal-02131156 \\ https://hal.science/hal-02131156}

Submitted on 15 Nov 2019

HAL is a multi-disciplinary open access archive for the deposit and dissemination of scientific research documents, whether they are published or not. The documents may come from teaching and research institutions in France or abroad, or from public or private research centers.
L'archive ouverte pluridisciplinaire HAL, est destinée au dépôt et à la diffusion de documents scientifiques de niveau recherche, publiés ou non, émanant des établissements d'enseignement et de recherche français ou étrangers, des laboratoires publics ou privés. 


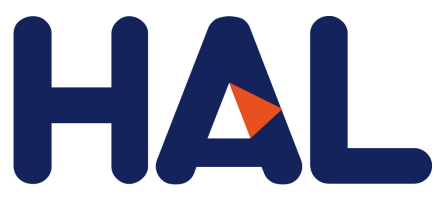

archives-ouvertes

\section{Perimeter-based coverage optimization to improve lifetime in wireless sensor networks}

Ali Kadhum Idrees, Karine Deschinkel, Michel Salomon, Raphael Couturier

\section{To cite this version:}

Ali Kadhum Idrees, Karine Deschinkel, Michel Salomon, Raphael Couturier. Perimeter-based coverage optimization to improve lifetime in wireless sensor networks. Engineering Optimization, 2016, 48 (11), pp.1951 - 1972. hal-02131156

\section{HAL Id: hal-02131156 \\ https://hal.archives-ouvertes.fr/hal-02131156}

Submitted on 15 Nov 2019

HAL is a multi-disciplinary open access archive for the deposit and dissemination of scientific research documents, whether they are published or not. The documents may come from teaching and research institutions in France or abroad, or from public or private research centers.
L'archive ouverte pluridisciplinaire HAL, est destinée au dépôt et à la diffusion de documents scientifiques de niveau recherche, publiés ou non, émanant des établissements d'enseignement et de recherche français ou étrangers, des laboratoires publics ou privés. 


\title{
Perimeter-based coverage optimization to improve lifetime in wireless sensor networks Ali Kadhum Idrees, Karine Deschinkel, Michel Salomon, Raphael Couturier
}

\begin{abstract}
Résumé
The most important problem in a Wireless Sensor Network (WSN) is to optimize the use of its limited energy provision, so that it can fulfill its monitoring task as long as possible. Among known available approaches that can be used to improve power management, lifetime coverage optimization provides activity scheduling which ensures sensing coverage while minimizing the energy cost. In this paper an approach called Perimeterbased Coverage Optimization protocol (PeCO) is proposed. It is a hybrid of centralized and distributed methods : the region of interest is first subdivided into subregions and the protocol is then distributed among sensor nodes in each subregion. The novelty of the approach lies essentially in the formulation of a new mathematical optimization model based on the perimeter coverage level to schedule sensors' activities. Extensive simulation experiments demonstrate that $\mathrm{PeCO}$ can offer longer lifetime coverage for WSNs compared to other protocols.
\end{abstract}

\section{Introduction}

The continuous progress in Micro Electro-Mechanical Systems (MEMS) and wireless communication hardware has given rise to the opportunity of using large networks of tiny sensors, called Wireless Sensor Networks (WSN) [1, 26], to fulfill monitoring tasks. A WSN consists of small low-powered sensors working together by communicating with one another through multi-hop radio communications. Each node can send the data it collects in its environment, thanks to its sensor, to the user by means of sink nodes. The features of a WSN makes it suitable for a wide range of applications in areas such as business, environment, health, industry, military, and so on [41]. Typically, a sensor node contains three main components [2] : a sensing unit able to measure physical, chemical, or biological phenomena observed in the environment; a processing unit which will process and store the collected measurements; a radio communication unit for data transmission and reception.

The energy needed by an active sensor node to perform sensing, processing, and communication is provided by a power supply which is a battery. This battery has a limited energy provision and it may be unsuitable or impossible to replace or recharge in most applications. Therefore it is necessary to deploy WSN with high density in order to increase reliability and to exploit node redundancy thanks to energy-efficient activity scheduling approaches. Indeed, the overlap of sensing areas can be exploited to schedule alternatively some sensors in a low power sleep mode and thus save energy. Overall, the main question that must be answered is : how is it possible to extend the lifetime coverage of a WSN as long as possible while ensuring a high level of coverage? These past few years many energy-efficient mechanisms have been suggested to retain energy and extend the lifetime of the WSNs [29].

This paper makes the following contributions :

1. A framework is devised to schedule nodes to be activated alternatively such that the network lifetime is prolonged while ensuring that a certain 
level of coverage is preserved. A key idea in the proposed framework is to exploit spatial and temporal subdivision. On the one hand, the area of interest is divided into several smaller subregions and, on the other hand, the time line is divided into periods of equal length. In each subregion the sensor nodes will cooperatively choose a leader which will schedule nodes' activities, and this grouping of sensors is similar to typical cluster architecture.

2. A new mathematical optimization model is proposed. Instead of trying to cover a set of specified points/targets as in most of the methods proposed in the literature, a mixed-integer program based on the perimeter coverage of each sensor is formulated. The model involves integer variables to capture the deviations between the actual level of coverage and the required level. Hence, an optimal schedule will be obtained by minimizing a weighted sum of these deviations.

3. Extensive simulation experiments are conducted using the discrete event simulator OMNeT ++ , to demonstrate the efficiency of the PeCO protocol. The PeCO protocol has been compared to two approaches found in the literature : DESK [33] and GAF [36], and also to the protocol DiLCO published in [15]. DiLCO uses the same framework as PeCO but is based on another optimization model for sensor scheduling.

The rest of the paper is organized as follows. In the next section some related work in the field is reviewed. Section 3 is devoted to the PeCO protocol description and Section 4 focuses on the coverage model formulation which is used to schedule the activation of sensor nodes. Section 5 presents simulations results and discusses the comparison with other approaches. Finally, concluding remarks are drawn and some suggestions are given for future works in Section 6.

\section{Related Literature}

This section summarizes some related works regarding the coverage problem and presents specific aspects of the $\mathrm{PeCO}$ protocol common with other literature works.

The most discussed coverage problems in literature can be classified in three categories [20] according to their respective monitoring objective. Hence, area coverage [24] means that every point inside a fixed area must be monitored, while target coverage [38] refers to the objective of coverage for a finite number of discrete points called targets, and barrier coverage $[11,17]$ focuses on preventing intruders from entering into the region of interest. In [8] authors transform the area coverage problem into the target coverage one, taking into account the intersection points among disks of sensors nodes or between disks of sensor nodes and boundaries. In [12] authors prove that if the perimeters of the sensors are sufficiently covered it will be the case for the whole area. They provide an algorithm in $O(n d \log d)$ time to compute the perimeter-coverage of each sensor. $d$ denotes the maximum number of sensors that are neighbors to a sensor, and 
$n$ is the total number of sensors in the network. In PeCO protocol, instead of determining the level of coverage of a set of discrete points, the optimization model is based on checking the perimeter-coverage of each sensor to activate a minimal number of sensors.

The major approach to extend network lifetime while preserving coverage is to divide/organize the sensors into a suitable number of set covers (disjoint or non-disjoint) [34], where each set completely covers a region of interest, and to successively activate these set covers. The network activity can be planned in advance and scheduled for the entire network lifetime or organized in periods, and the set of active sensor nodes decided at the beginning of each period [22]. In fact, many authors propose algorithms working in such a periodic fashion $[32,37$, 25]. Active node selection is determined based on the problem requirements (e.g. area monitoring, connectivity, or power efficiency). For instance, [16] address the problem of maximizing the lifetime by dividing sensors into the maximum number of disjoint subsets such that each subset can ensure both coverage and connectivity. A greedy algorithm is applied once to solve this problem and the computed sets are activated in succession to achieve the desired network lifetime. Motivated by these works, PeCO protocol works in periods, where each period contains a preliminary phase for information exchange and decisions, followed by a sensing phase where one cover set is in charge of the sensing task.

Various centralized and distributed approaches, or even a mixing of these two concepts, have been proposed to extend the network lifetime [43]. In distributed algorithms $[33,28,39]$ each sensor decides of its own activity scheduling after an information exchange with its neighbors. The main interest of such an approach is to avoid long range communications and thus to reduce the energy dedicated to the communications. Unfortunately, since each node has information on its immediate neighbors only (usually the one-hop ones), it may make a bad decision leading to a global suboptimal solution. Conversely, centralized algorithms [4, 44,27 always provide nearly optimal solutions since the algorithm has a global view of the whole network. The disadvantage of a centralized method is obviously its high cost in communications needed to transmit to a single node, the base station which will globally schedule nodes' activities, data from all the other sensor nodes in the area. The price in communications can be huge since long range communications will be needed. In fact the larger the WSN, the higher the communication energy cost. In order to be suitable for large-scale networks, in the PeCO protocol the area of interest is divided into several smaller subregions, and in each one, a node called the leader is in charge of selecting the active sensors for the current period. Thus the PeCO protocol is scalable and a globally distributed method, whereas it is centralized in each subregion.

Various coverage scheduling algorithms have been developed these past few years. Many of them, dealing with the maximization of the number of cover sets, are heuristics. These heuristics involve the construction of a cover set by including in priority the sensor nodes which cover critical targets, that is to say targets that are covered by the smallest number of sensors [3, 44]. Other approaches are based on mathematical programming formulations [5, 35, 27, 40] and dedicated techniques (solving with a branch-and-bound algorithm available 
in optimization solver). The problem is formulated as an optimization problem (maximization of the lifetime or number of cover sets) under target coverage and energy constraints. Column generation techniques, well-known and widely practiced techniques for solving linear programs with too many variables, have also been used [6, 30, 9]. In the PeCO protocol, each leader, in charge of a subregion, solves an integer program which has a twofold objective : minimizing the overcoverage and the undercoverage of the perimeter of each sensor.

The authors in [15] propose a Distributed Lifetime Coverage Optimization (DiLCO) protocol, which maintains the coverage and improves the lifetime in WSNs. It is an improved version of a research work presented in [14]. First, the area of interest is partitioned into subregions using a divide-and-conquer method. The DiLCO protocol is then distributed on the sensor nodes in each subregion in a second step. Hence this protocol combines two techniques : a leader election in each subregion, followed by an optimization-based node activity scheduling performed by each elected leader. The proposed DiLCO protocol is a periodic protocol where each period is decomposed into 4 phases : information exchange, leader election, decision, and sensing. The simulations show that DiLCO is able to increase the WSN lifetime and provides improved coverage performance. In the PeCO protocol, a new mathematical optimization model is proposed. Instead of trying to cover a set of specified points/targets as in the DiLCO protocol, an integer program based on the perimeter coverage of each sensor is formulated. The model involves integer variables to capture the deviations between the actual level of coverage and the required level. The idea is that an optimal scheduling will be obtained by minimizing a weighted sum of these deviations.

\section{The PECO Protocol Description}

\subsection{Assumptions and Models}

A WSN consisting of $J$ stationary sensor nodes randomly and uniformly distributed in a bounded sensor field is considered. The wireless sensors are deployed in high density to ensure initially a high coverage ratio of the area of interest. All the sensor nodes are supposed to be homogeneous in terms of communication, sensing, and processing capabilities and heterogeneous from the energy provision point of view. The location information is available to a sensor node either through hardware such as embedded GPS or location discovery algorithms. A Boolean disk coverage model, which is the most widely used sensor coverage model in the literature, is considered and all sensor nodes have a constant sensing range $R_{s}$. Thus, all the space points within a disk centered at a sensor with a radius equal to the sensing range are said to be covered by this sensor. The communication range $R_{c}$ is assumed to satisfy: $R_{c} \geq 2 \cdot R_{s}$. In fact, [42] proved that if the transmission range fulfills the previous hypothesis, the complete coverage of a convex area implies connectivity among active nodes.

The PeCO protocol uses the same perimeter-coverage model as [12]. It can 
be expressed as follows : a sensor is said to be perimeter covered if all the points on its perimeter are covered by at least one sensor other than itself. Authors [12] proved that a network area is $k$-covered (every point in the area is covered by at least $k$ sensors) if and only if each sensor in the network is $k$-perimeter-covered (perimeter covered by at least $k$ sensors).

Figure 1(a) shows the coverage of sensor node 0 . On this figure, sensor 0 has nine neighbors. For each neighbor the two points resulting from the intersection of the two sensing areas have been reported on its perimeter (the perimeter of the disk covered by the sensor 0). These points are denoted for neighbor $i$ by $i L$ and $i R$, respectively for left and right from a neighboring point of view. The resulting couples of intersection points subdivide the perimeter of sensor 0 into portions called arcs.

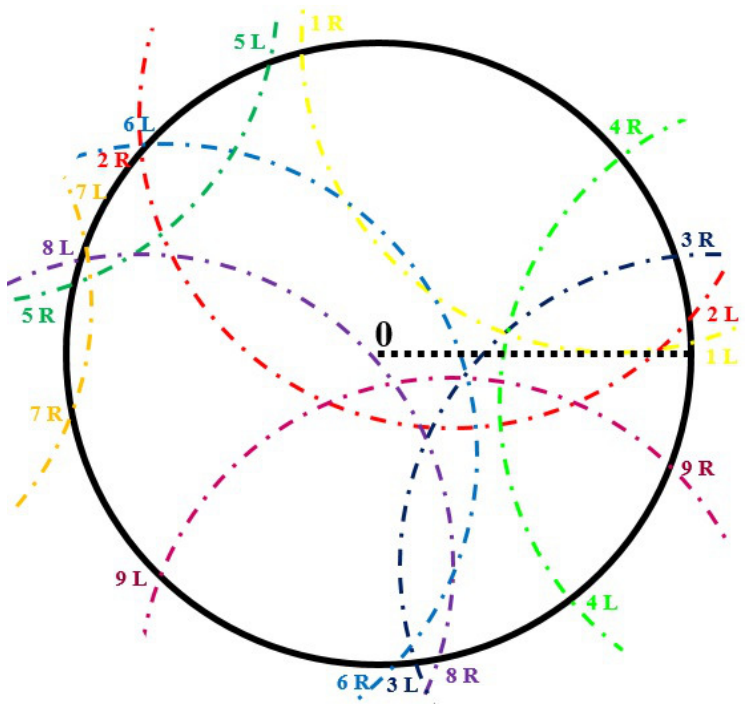

(a)

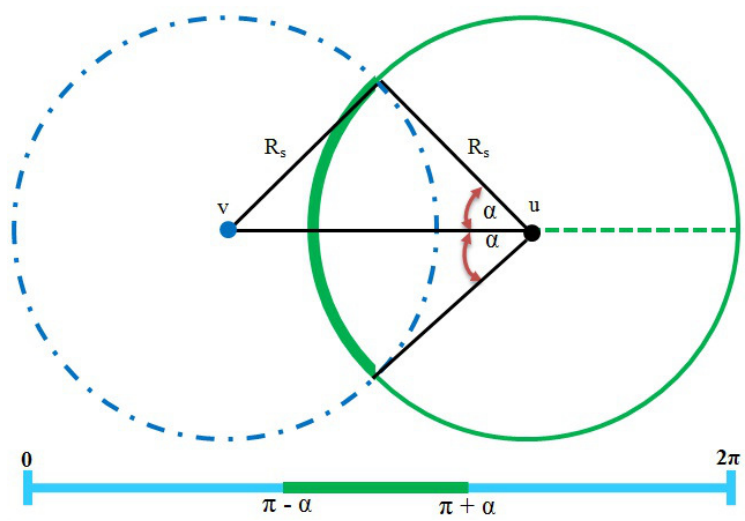

(b)

Figure 1 - (a) Perimeter coverage of sensor node 0 and (b) finding the arc of $u$ 's perimeter covered by $v$. 
Figure 1(b) describes the geometric information used to find the locations of the left and right points of an arc on the perimeter of a sensor node $u$ covered by a sensor node $v$. Node $v$ is supposed to be located on the west side of sensor $u$, with the following respective coordinates in the sensing area : $\left(v_{x}, v_{y}\right)$ and $\left(u_{x}, u_{y}\right)$. From the previous coordinates the euclidean distance between nodes $u$ and $v$ is computed as follows :

$$
\operatorname{Dist}(u, v)=\sqrt{\left(u_{x}-v_{x}\right)^{2}+\left(u_{y}-v_{y}\right)^{2}},
$$

while the angle $\alpha$ is obtained through the formula :

$$
\alpha=\arccos \left(\frac{\operatorname{Dist}(u, v)}{2 R_{s}}\right) .
$$

The arc on the perimeter of $u$ defined by the angular interval $[\pi-\alpha, \pi+\alpha]$ is then said to be perimeter-covered by sensor $v$.

Every couple of intersection points is placed on the angular interval $[0,2 \pi)$ in a counterclockwise manner, leading to a partitioning of the interval. Figure 1(a) illustrates the arcs for the nine neighbors of sensor 0 and Table 1 gives the position of the corresponding arcs in the interval $[0,2 \pi)$. More precisely, the points are ordered according to the measures of the angles defined by their respective positions. The intersection points are then visited one after another, starting from the first intersection point after point zero, and the maximum level of coverage is determined for each interval defined by two successive points. The maximum level of coverage is equal to the number of overlapping arcs. For example, between $5 L$ and $6 L$ the maximum level of coverage is equal to 3 (the value is given at the bottom of Figure 2), which means that at most 2 neighbors can cover the perimeter in addition to node 0 . Table 1 summarizes for each coverage interval the maximum level of coverage and the sensor nodes covering the perimeter. The example discussed above is thus given by the sixth line of the table.

In the PeCO protocol, the scheduling of the sensor nodes' activities is formulated with a mixed-integer program based on coverage intervals [13]. The formulation of the coverage optimization problem is detailed in Section 4. Note that when a sensor node has a part of its sensing range outside the WSN sensing field, as in Figure 3, the maximum coverage level for this arc is set to $\infty$ and the corresponding interval will not be taken into account by the optimization algorithm.

\subsection{Main Idea}

The WSN area of interest is, in a first step, divided into regular homogeneous subregions using a divide-and-conquer algorithm. In a second step the protocol will be executed in a distributed way in each subregion simultaneously to schedule nodes' activities for one sensing period. Sensor nodes are assumed to be deployed almost uniformly over the region. The regular subdivision is made such that the number of hops between any pairs of sensors inside a subregion is less than or equal to 3 . 
TABLE 1 - Coverage intervals and contributing sensors for node 0

\begin{tabular}{|c|c|c|c|c|c|c|c|c|}
\hline $\begin{array}{c}\text { Left } \\
\text { point } \\
\text { angle } \alpha\end{array}$ & $\begin{array}{c}\text { Interval } \\
\text { left } \\
\text { point }\end{array}$ & $\begin{array}{c}\text { Interval } \\
\text { right } \\
\text { point }\end{array}$ & $\begin{array}{c}\text { Maximum } \\
\text { coverage } \\
\text { level }\end{array}$ & \multicolumn{6}{|c|}{$\begin{array}{c}\text { Set of sensors } \\
\text { involved }\end{array}$} \\
\hline 0.0291 & $1 \mathrm{~L}$ & $2 \mathrm{~L}$ & 4 & 0 & 1 & 3 & 4 & \\
\hline 0.104 & $2 \mathrm{~L}$ & $3 \mathrm{R}$ & 5 & 0 & 1 & 3 & 4 & 2 \\
\hline 0.3168 & $3 \mathrm{R}$ & $4 \mathrm{R}$ & 4 & 0 & 1 & 4 & 2 & \\
\hline 0.6752 & $4 \mathrm{R}$ & $1 \mathrm{R}$ & 3 & 0 & 1 & 2 & & \\
\hline 1.8127 & $1 \mathrm{R}$ & $5 \mathrm{~L}$ & 2 & 0 & 2 & & & \\
\hline 1.9228 & $5 \mathrm{~L}$ & $6 \mathrm{~L}$ & 3 & 0 & 2 & 5 & & \\
\hline 2.3959 & $6 \mathrm{~L}$ & $2 \mathrm{R}$ & 4 & 0 & 2 & 5 & 6 & \\
\hline 2.4258 & $2 \mathrm{R}$ & $7 \mathrm{~L}$ & 3 & 0 & 5 & 6 & & \\
\hline 2.7868 & $7 \mathrm{~L}$ & $8 \mathrm{~L}$ & 4 & 0 & 5 & 6 & 7 & \\
\hline 2.8358 & $8 \mathrm{~L}$ & $5 \mathrm{R}$ & 5 & 0 & 5 & 6 & 7 & 8 \\
\hline 2.9184 & $5 \mathrm{R}$ & $7 \mathrm{R}$ & 4 & 0 & 6 & 7 & 8 & \\
\hline 3.3301 & $7 \mathrm{R}$ & $9 \mathrm{R}$ & 3 & 0 & 6 & 8 & & \\
\hline 3.9464 & $9 \mathrm{R}$ & $6 \mathrm{R}$ & 4 & 0 & 6 & 8 & 9 & \\
\hline 4.767 & $6 \mathrm{R}$ & $3 \mathrm{~L}$ & 3 & 0 & 8 & 9 & & \\
\hline 4.8425 & $3 \mathrm{~L}$ & $8 \mathrm{R}$ & 4 & 0 & 3 & 8 & 9 & \\
\hline 4.9072 & $8 \mathrm{R}$ & $4 \mathrm{~L}$ & 3 & 0 & 3 & 9 & & \\
\hline 5.3804 & $4 \mathrm{~L}$ & $9 \mathrm{R}$ & 4 & 0 & 3 & 4 & 9 & \\
\hline 5.9157 & $9 \mathrm{R}$ & $1 \mathrm{~L}$ & 3 & 0 & 3 & 4 & & \\
\hline & & & & & & & \\
\hline
\end{tabular}




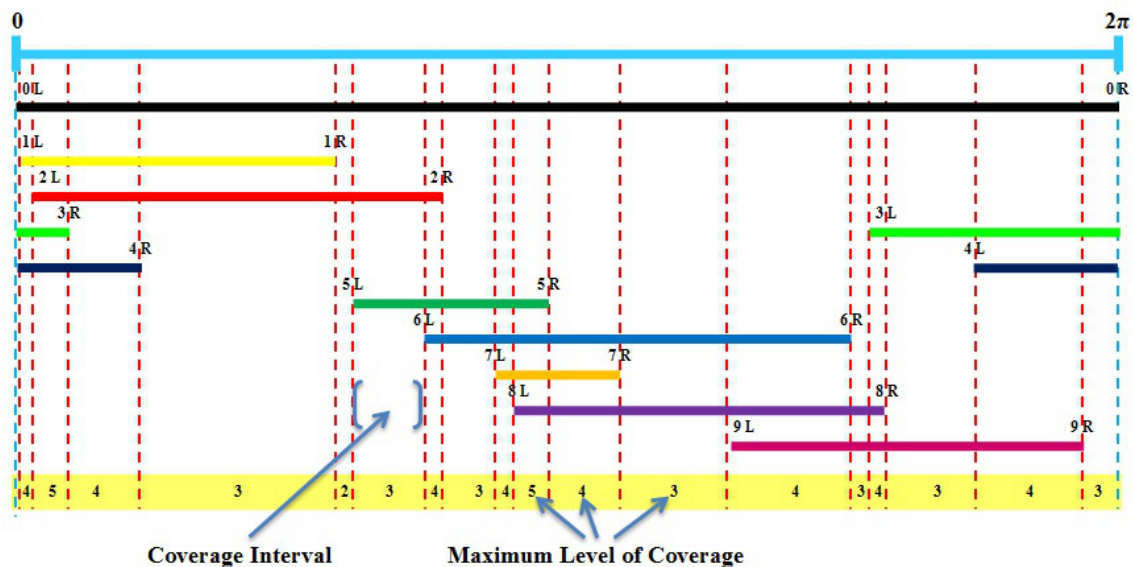

FiguRE 2 - Maximum coverage levels for perimeter of sensor node 0.

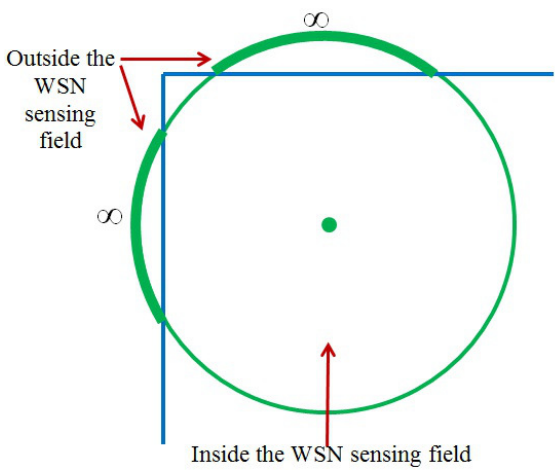

FIGURE 3 - Sensing range outside the WSN's area of interest.

As shown in Figure 4, node activity scheduling is produced by the proposed protocol in a periodic manner. Each period is divided into 4 stages : Information (INFO) Exchange, Leader Election, Decision (the result of an optimization problem), and Sensing. For each period there is exactly one set cover responsible for the sensing task. Protocols based on a periodic scheme, like PeCO, are more robust against an unexpected node failure. On the one hand, if a node failure is discovered before taking the decision, the corresponding sensor node will not be considered by the optimization algorithm. On the other hand, if the sensor failure happens after the decision, the sensing task of the network will be temporarily affected : only during the period of sensing until a new period starts, since a new set cover will take charge of the sensing task in the next period. The energy consumption and some other constraints can easily be taken into account since the sensors can update and then exchange their information (including their residual energy) at the beginning of each period. However, the 


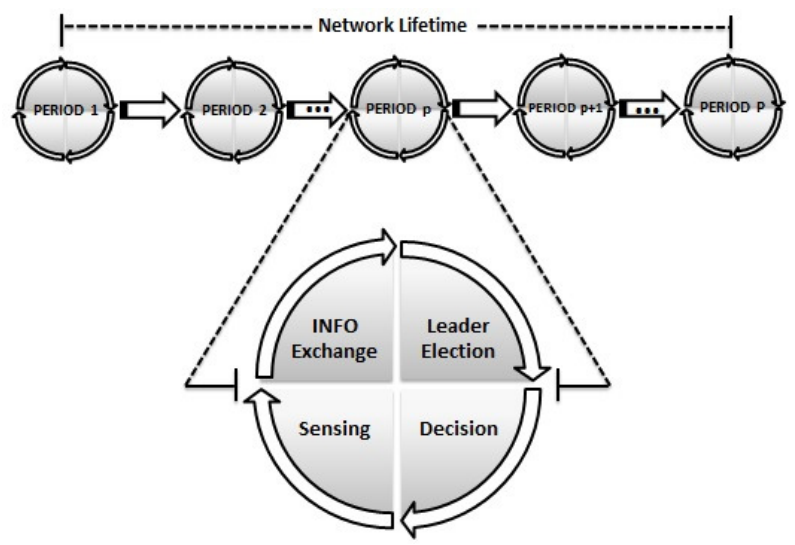

Figure 4 - PeCO protocol.

pre-sensing phases (INFO Exchange, Leader Election, and Decision) are energy consuming, even for nodes that will not join the set cover to monitor the area. Sensing period duration is adapted according to the QoS requirements of the application.

Two types of packets used by the PeCO protocol are defined :

- INFO packet : sent by each sensor node to all the nodes inside a same subregion for information exchange.

- ActiveSleep packet : sent by the leader to all the nodes in its subregion to transmit to them their respective status (stay Active or go Sleep) during the sensing phase.

Five statuses are possible for a sensor node in the network :

- LISTENING : waits for a decision (to be active or not);

- COMPUTATION : executes the optimization algorithm as leader to determine the activities scheduling;

- ACTIVE : node is sensing;

- SLEEP : node is turned off;

- COMMUNICATION : transmits or receives packets.

\subsection{PeCO Protocol Algorithm}

The pseudocode implementing the protocol on a node is given below. More precisely, Algorithm 1 gives a brief description of the protocol applied by a sensor node $s_{k}$ where $k$ is the node index in the WSN.

In this algorithm, K.CurrentSize and K.PreviousSize respectively represent the current number and the previous number of living nodes in the subnetwork of the subregion. At the beginning of the first period K.PreviousSize is initialized to zero. Initially, the sensor node checks its remaining energy $R E_{k}$, which must be greater than a threshold $E_{t h}$ in order to participate in the current period. Each sensor node determines its position and its subregion using 


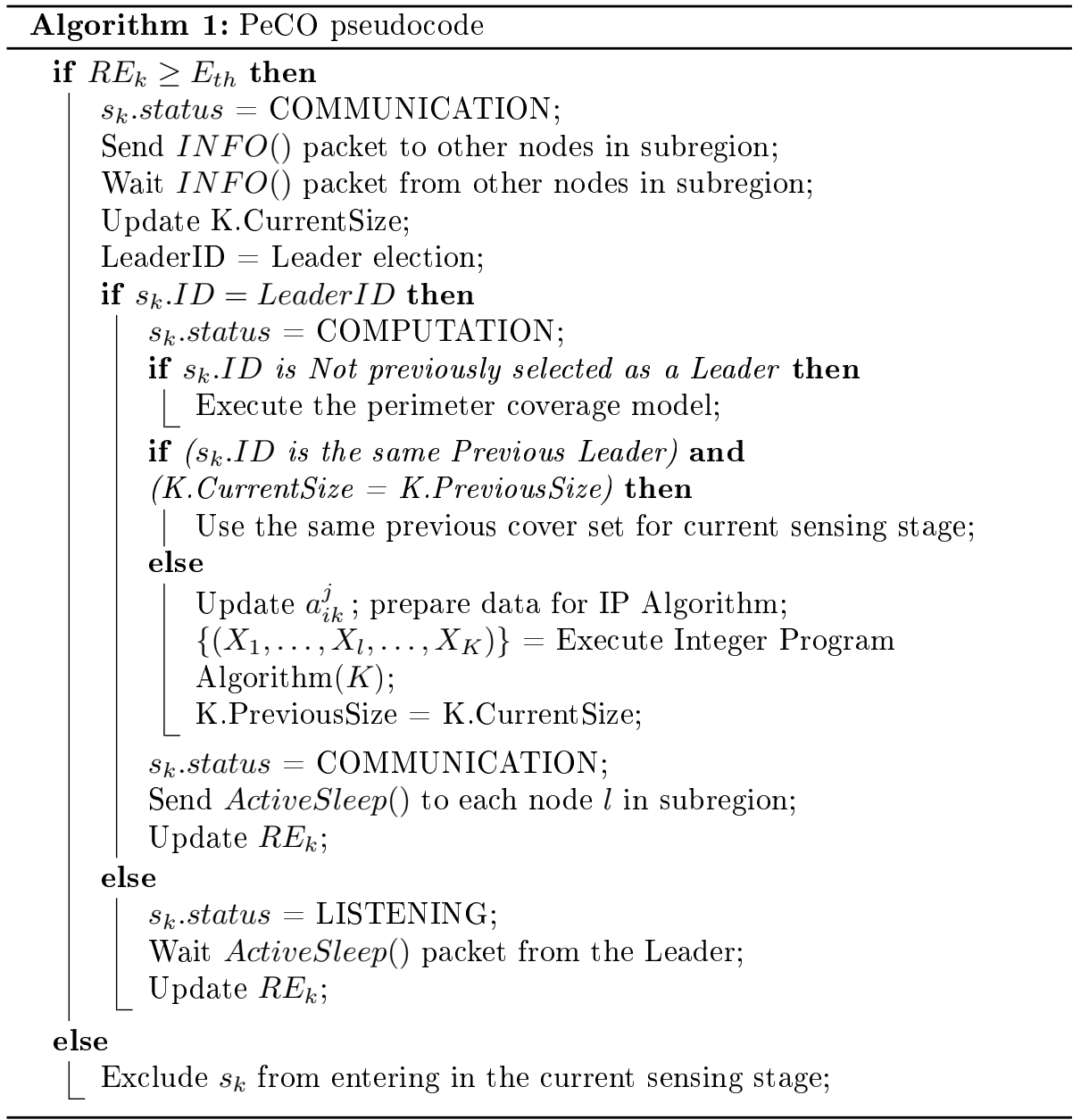


an embedded GPS or a location discovery algorithm. After that, all the sensors collect position coordinates, remaining energy, sensor node ID, and the number of their one-hop live neighbors during the information exchange. Both INFO packet and ActiveSleep packet contain two parts : header and data payload. The sensor ID is included in the header, where the header size is 8 bits. The data part includes position coordinates (64 bits), remaining energy (32 bits), and the number of one-hop live neighbors ( 8 bits). Therefore the size of the INFO packet is 112 bits. The ActiveSleep packet is 16 bits size, 8 bits for the header and 8 bits for data part that includes only sensor status ( 0 or 1$)$. The sensors inside a same region cooperate to elect a leader. The selection criteria for the leader are (in order of priority) :

1. larger number of neighbors ;

2. larger remaining energy;

3. and then, in case of equality, larger indexes.

Once chosen, the leader collects information to formulate and solve the integer program which allows to build the set of active sensors in the sensing stage.

\section{Perimeter-based Coverage Problem Formula- tion}

In this section, the perimeter-based coverage problem is mathematically formulated. It has been proved to be a NP-hard problem by [13]. Authors study the coverage of the perimeter of a large object requiring to be monitored. For the proposed formulation in this paper, the large object to be monitored is the sensor itself (or more precisely its sensing area).

The following notations are used throughout the section.

First, the following sets :

- $S$ represents the set of sensor nodes;

- $A \subseteq S$ is the subset of alive sensors;

- $I_{j}$ designates the set of coverage intervals (CI) obtained for sensor $j$.

$I_{j}$ refers to the set of coverage intervals which has been defined according to the method introduced in Subsection 3.1. For a coverage interval $i$, let $a_{i k}^{j}$ denote the indicator function of whether sensor $k$ is involved in coverage interval $i$ of sensor $j$, that is :

$$
a_{i k}^{j}= \begin{cases}1 & \begin{array}{l}
\text { if sensor } k \text { is involved in the } \\
\text { coverage interval } i \text { of sensor } j \\
0
\end{array} \\
\text { otherwise. }\end{cases}
$$

Note that $a_{i k}^{k}=1$ by definition of the interval.

Second, several variables are defined. Hence, each binary variable $X_{k}$ determines the activation of sensor $k$ in the sensing phase $\left(X_{k}=1\right.$ if the sensor $k$ is active or 0 otherwise). $M_{i}^{j}$ is a variable which measures the undercoverage for the coverage interval $i$ corresponding to sensor $j$. In the same way, the overcoverage for the same coverage interval is given by the variable $V_{i}^{j}$. 
To sustain a level of coverage equal to $l$ all along the perimeter of sensor $j$, at least $l$ sensors involved in each coverage interval $i \in I_{j}$ of sensor $j$ have to be active. According to the previous notations, the number of active sensors in the coverage interval $i$ of sensor $j$ is given by $\sum_{k \in A} a_{i k}^{j} X_{k}$. To extend the network lifetime, the objective is to activate a minimal number of sensors in each period to ensure the desired coverage level. As the number of alive sensors decreases, it becomes impossible to reach the desired level of coverage for all coverage intervals. Therefore variables $M_{i}^{j}$ and $V_{i}^{j}$ are introduced as a measure of the deviation between the desired number of active sensors in a coverage interval and the effective number. And these deviations are minimized, first to force the activation of a minimal number of sensors to ensure the desired coverage level, and if the desired level cannot be completely satisfied, to reach a coverage level as close as possible to the desired one.

The coverage optimization problem can then be mathematically expressed as follows :

$$
\text { Minimize } \sum_{j \in S} \sum_{i \in I_{j}}\left(\alpha_{i}^{j} M_{i}^{j}+\beta_{i}^{j} V_{i}^{j}\right)
$$

Subject to :

$$
\begin{aligned}
& \sum_{k \in A}\left(a_{i k}^{j} X_{k}\right)+M_{i}^{j} \geq l \quad \forall i \in I_{j}, \forall j \in S \\
& \sum_{k \in A}\left(a_{i k}^{j} X_{k}\right)-V_{i}^{j} \leq l \quad \forall i \in I_{j}, \forall j \in S \\
& X_{k} \in\{0,1\}, \forall k \in A \\
& M_{i}^{j}, V_{i}^{j} \in \mathbb{R}^{+}
\end{aligned}
$$

If a given level of coverage $l$ is required for one sensor, the sensor is said to be undercovered (respectively overcovered) if the level of coverage of one of its CI is less (respectively greater) than $l$. If the sensor $j$ is undercovered, there exists at least one of its CI (say $i$ ) for which the number of active sensors (denoted by $\left.l^{i}\right)$ covering this part of the perimeter is less than $l$ and in this case : $M_{i}^{j}=l-l^{i}$, $V_{i}^{j}=0$. Conversely, if the sensor $j$ is overcovered, there exists at least one of its CI (say $i$ ) for which the number of active sensors (denoted by $l^{i}$ ) covering this part of the perimeter is greater than $l$ and in this case $: M_{i}^{j}=0, V_{i}^{j}=l^{i}-l$.

$\alpha_{i}^{j}$ and $\beta_{i}^{j}$ are nonnegative weights selected according to the relative importance of satisfying the associated level of coverage. For example, weights associated with coverage intervals of the specified part of a region may be given by a relatively larger magnitude than weights associated with another region. This kind of mixed-integer program is inspired from the model developed for brachytherapy treatment planning to optimize dose distribution [19]. The choice of the values for variables $\alpha$ and $\beta$ should be made according to the needs of the application. $\alpha$ should be large enough to prevent undercoverage and so to reach the highest possible coverage ratio. $\beta$ should be large enough to prevent overcoverage and so to activate a minimum number of sensors. The mixed-integer program must be solved by the leader in each subregion at the beginning of 
each sensing phase, whenever the environment has changed (new leader, death of some sensors). Note that the number of constraints in the model is constant (constraints of coverage expressed for all sensors), whereas the number of variables $X_{k}$ decreases over periods, since only alive sensors (sensors with enough energy to be alive during one sensing phase) are considered in the model.

\section{Performance Evaluation and Analysis}

\subsection{Simulation Settings}

The WSN area of interest is supposed to be divided into 16 regular subregions and the energy consumption model used is described in previous work [15]. Table 2 gives the chosen parameters settings.

\begin{tabular}{c|c} 
TABLE $2-$ Relevant parameters for network initialization \\
\hline Parameter & Value \\
\hline Sensing field & $(50 \times 25) \mathrm{m}^{2}$ \\
WSN size & $100,150,200,250$, and 300 nodes \\
Initial energy & in range 500-700 Joules \\
Sensing period & duration of 60 minutes \\
$E_{t h}$ & 36 Joules \\
$R_{s}$ & $5 \mathrm{~m}$ \\
$R_{c}$ & $10 \mathrm{~m}$ \\
$\alpha_{i}^{j}$ & 0.6 \\
$\beta_{i}^{j}$ & 0.4
\end{tabular}

To obtain experimental results which are relevant, simulations with five different node densities going from 100 to 300 nodes were performed considering each time 25 randomly generated networks. The nodes are deployed on a field of interest of $(50 \times 25) \mathrm{m}^{2}$ in such a way that they cover the field with a high coverage ratio. Each node has an initial energy level, in Joules, which is randomly drawn in the interval [500 - 700]. If its energy provision reaches a value below the threshold $E_{t h}=36$ Joules, the minimum energy needed for a node to stay active during one period, it will no longer participate in the coverage task. This value corresponds to the energy needed by the sensing phase, obtained by multiplying the energy consumed in the active state $(9.72 \mathrm{~mW})$ with the time in seconds for one period (3600 seconds), and adding the energy for the pre-sensing phases. According to the interval of initial energy, a sensor may be active during at most 20 periods. the information exchange to update the coverage is executed every hour, but the length of the sensing period could be reduced and adapted dynamically. On the one hand a small sensing period would allow the network to be more reliable but would have resulted in higher communication costs. On the other hand the choice of a long duration may cause problems in case of nodes failure during the sensing period. 
The values of $\alpha_{i}^{j}$ and $\beta_{i}^{j}$ have been chosen to ensure a good network coverage and a longer WSN lifetime. Higher priority is given to the undercoverage (by setting the $\alpha_{i}^{j}$ with a larger value than $\beta_{i}^{j}$ ) so as to prevent the non-coverage for the interval $i$ of the sensor $j$. On the other hand, $\beta_{i}^{j}$ is assigned to a value which is slightly lower so as to minimize the number of active sensor nodes which contribute in covering the interval. Subsection 5.2.6 investigates more deeply how the values of both parameters affect the performance of the PeCO protocol.

The following performance metrics are used to evaluate the efficiency of the approach.

- Network Lifetime : the lifetime is defined as the time elapsed until the coverage ratio falls below a fixed threshold. Lifetime 95 and Lifetime L $_{50}$ denote, respectively, the amount of time during which is guaranteed a level of coverage greater than $95 \%$ and $50 \%$. The WSN can fulfill the expected monitoring task until all its nodes have depleted their energy or if the network is no more connected. This last condition is crucial because without network connectivity a sensor may not be able to send to a base station an event it has sensed.

- Coverage Ratio (CR) : it measures how well the WSN is able to observe the area of interest. Here the sensor field is discretized as a regular grid, which yields the following equation :

$$
\mathrm{CR}(\%)=\frac{n}{N} \times 100
$$

where $n$ is the number of covered grid points by active sensors of every subregions during the current sensing phase and $N$ is total number of grid points in the sensing field. A layout of $N=51 \times 26=1326$ grid points is considered in the simulations.

- Active Sensors Ratio (ASR) : a major objective of the proposed protocol is to activate as few nodes as possible, in order to minimize the communication overhead and maximize the WSN lifetime. The active sensors ratio is defined as follows :

$$
\operatorname{ASR}(\%)=\frac{\sum_{r=1}^{R}\left|A_{r}^{p}\right|}{|S|} \times 100
$$

where $\left|A_{r}^{p}\right|$ is the number of active sensors in the subregion $r$ in the sensing period $p, R$ is the number of subregions, and $|J|$ is the number of sensors in the network.

- Energy Saving Ratio (ESR) : this metric, which shows the ability of a protocol to save energy, is defined by :

$$
\operatorname{ESR}(\%)=\frac{\text { Number of alive sensors during this round }}{\text { Total number of sensors in the network }} \times 100 .
$$

- Energy Consumption (EC) : energy consumption can be seen as the total energy consumed by the sensors during Lifetime $_{95}$ or Lifetime $_{50}$, 
divided by the number of periods. The value of EC is computed according to this formula :

$$
\mathrm{EC}=\frac{\sum_{p=1}^{P}\left(E_{p}^{\mathrm{com}}+E_{p}^{\mathrm{list}}+E_{p}^{\mathrm{comp}}+E_{p}^{a}+E_{p}^{s}\right)}{P},
$$

where $P$ corresponds to the number of periods. The total energy consumed by the sensors comes through taking into consideration four main energy factors. The first one, denoted $E_{p}^{\text {com }}$, represents the energy consumption spent by all the nodes for wireless communications during period $p . E_{p}^{\text {list }}$, the next factor, corresponds to the energy consumed by the sensors in LISTENING status before receiving the decision to go active or sleep in period $p . E_{p}^{\text {comp }}$ refers to the energy needed by all the leader nodes to solve the integer program during a period (COMPUTATION status). Finally, $E_{p}^{a}$ and $E_{p}^{s}$ indicate the energy consumed by the WSN during the sensing phase (active and sleeping nodes).

\subsection{Simulation Results}

The PeCO protocol has been implemented in OMNeT $++[31]$ simulator in order to assess and analyze its performance. The simulations were run on a DELL laptop with an Intel Core i3 $2370 \mathrm{M}(1.8 \mathrm{GHz})$ processor (2 cores) whose MIPS (Million Instructions Per Second) rate is equal to 35330 . To be consistent with the use of a sensor node based on Atmels AVR ATmega103L microcontroller $(6 \mathrm{MHz})$ having a MIPS rate equal to 6 , the original execution time on the laptop is multiplied by $2944.2\left(\frac{35330}{2} \times \frac{1}{6}\right)$. Energy consumption is calculated according to the power consumption values, in milliWatt per second, given in Table 3, based on the energy model proposed in [33].

TABle 3 - Power consumption values

\begin{tabular}{|c|c|c|c|c|}
\hline Sensor status & $\mathrm{MCU}$ & Radio & Sensing & Power $(\mathrm{mW})$ \\
\hline LISTENING & On & On & On & 20.05 \\
\hline ACTIVE & On & Off & On & 9.72 \\
\hline SLEEP & Off & Off & Off & 0.02 \\
\hline COMPUTATION & On & On & On & 26.83 \\
\hline
\end{tabular}

The modeling language for Mathematical Programming (AMPL) [10] is used to generate the integer program instance in a standard format, which is then read and solved by the optimization solver GLPK (GNU Linear Programming Kit available in the public domain) [23] through a Branch-and-Bound method. In practice, executing GLPK on a sensor node is obviously intractable due to the huge memory use. Fortunately, to solve the optimization problem, the use of commercial solvers like CPLEX [7] which are less memory consuming and more 
efficient is possible, or a lightweight heuristic may be implemented. For example, for a WSN of 200 sensor nodes, a leader node has to deal with constraints induced by about 12 sensor nodes. In that case, to solve the optimization problem a memory consumption of more than $1 \mathrm{MB}$ can be observed with GLPK, whereas less than $300 \mathrm{~KB}$ would be needed with CPLEX.

Besides PeCO, three other protocols will be evaluated for comparison purposes. The first one, called DESK, is a fully distributed coverage algorithm proposed by [33]. The second one, called GAF [36], consists in dividing the monitoring area into fixed squares. Then, during the decision phase, in each square, one sensor is chosen to remain active during the sensing phase. The last one, the DiLCO protocol [15], is an improved version of a research work presented in [14]. PeCO and DiLCO protocols are based on the same framework. In particular, the choice for the simulations of a partitioning in 16 subregions was made because it corresponds to the configuration producing the best results for DiLCO. Of course, this number of subregions should be adapted according to the size of the area of interest and the number of sensors. The protocols are distinguished from one another by the formulation of the integer program providing the set of sensors which have to be activated in each sensing phase. The DiLCO protocol tries to satisfy the coverage of a set of primary points, whereas the objective of the $\mathrm{PeCO}$ protocol is to reach a desired level of coverage for each sensor perimeter. In the experimentations, a level of coverage equal to one $(l=1)$ is chosen .

\subsubsection{Coverage Ratio}

Figure 5 shows the average coverage ratio for 200 deployed nodes obtained with the four protocols. DESK, GAF, and DiLCO provide a slightly better coverage ratio with respectively $99.99 \%, 99.91 \%$, and $99.02 \%$, compared to the 98.76\% produced by PeCO for the first periods. This is due to the fact that at the beginning the DiLCO and PeCO protocols put more redundant sensors to sleep status (which slightly decreases the coverage ratio), while the two other protocols activate more sensor nodes. Later, when the number of periods is beyond 70 , it clearly appears that $\mathrm{PeCO}$ provides a better coverage ratio and keeps a coverage ratio greater than $50 \%$ for longer periods ( 15 more compared to DiLCO, 40 more compared to DESK). The energy saved by PeCO in the early periods allows later a substantial increase of the coverage performance.

\subsubsection{Active Sensors Ratio}

Minimizing the number of active sensor nodes in each period is essential to minimize the energy consumption and thus to maximize the network lifetime. Figure 6 shows the average active nodes ratio for 200 deployed nodes. DESK and GAF have $30.36 \%$ and $34.96 \%$ active nodes for the first fourteen rounds, and the DiLCO and PeCO protocols compete perfectly with only $17.92 \%$ and $20.16 \%$ active nodes during the same time interval. As the number of periods increases, the PeCO protocol has a lower number of active nodes in comparison 


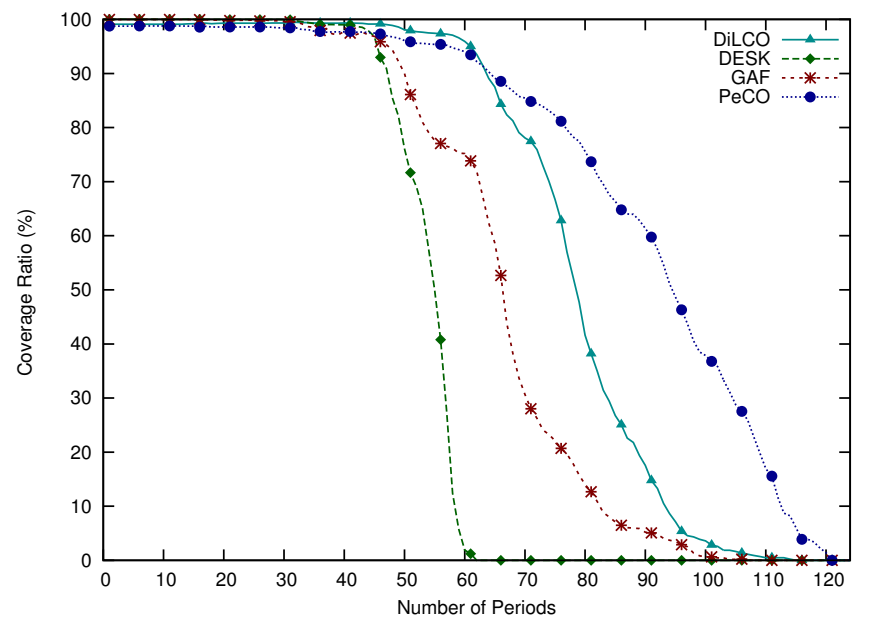

FigURE 5 - Coverage ratio for 200 deployed nodes.

with the three other approaches and exhibits a slow decrease, while keeping a greater coverage ratio as shown in Figure 5.

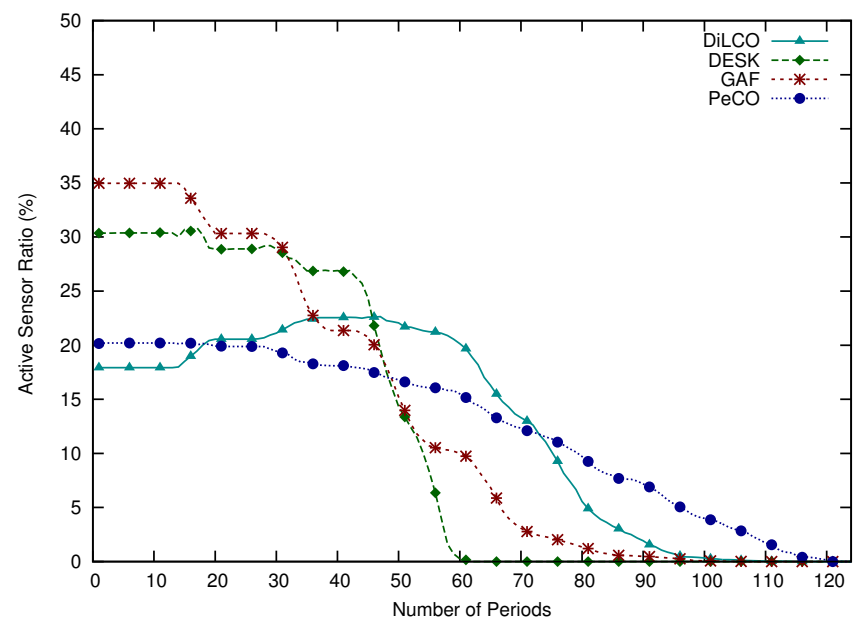

Figure 6 - Active sensors ratio for 200 deployed nodes.

\subsubsection{Energy Saving Ratio}

The simulation results show that the protocol PeCO saves efficiently energy by turning off some sensors during the sensing phase. As shown in Figure 7, GAF provides better energy saving than $\mathrm{PeCO}$ for the first fifty rounds. Indeed GAF balances the energy consumption among sensor nodes inside each small fixed 
grid and thus permits to extend the life of sensors in each grid fairly. However, at the same time it turns on a large number of sensors and that leads later to quickly deplete sensor's batteries. DESK algorithm shows less energy saving compared with other approaches. In comparison with PeCO, DiLCO protocol usually provides lower energy saving ratios. Moreover, it can be noticed that after round fifty, PeCO protocol exhibits the slowest decrease among all the considered protocols.

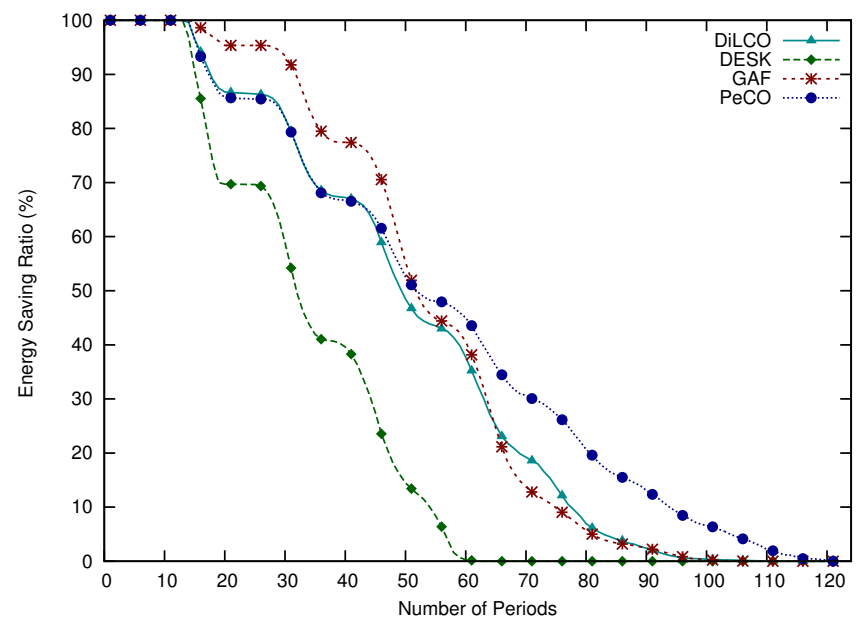

Figure 7 - Energy Saving Ratio for 200 deployed nodes.

\subsubsection{Energy Consumption}

The effect of the energy consumed by the WSN during the communication, computation, listening, active, and sleep status is studied for different network densities and the four approaches compared. Figures 8(a) and (b) illustrate the energy consumption for different network sizes and for Lifetime 95 and Lifetime $_{50}$. The results show that the PeCO protocol is the most competitive from the energy consumption point of view. As shown by both figures, PeCO consumes much less energy than the other methods. One might think that the resolution of the integer program is too costly in energy, but the results show that it is very beneficial to lose a bit of time in the selection of sensors to activate. Indeed the optimization program allows to reduce significantly the number of active sensors and also the energy consumption while keeping a good coverage level. The energy overhead when increasing network size is the lowest with PeCO.

\subsubsection{Network Lifetime}

In comparison with the two other approaches, PeCO and DiLCO protocols are better for prolonging the network lifetime. In Figures 9(a) and (b), 


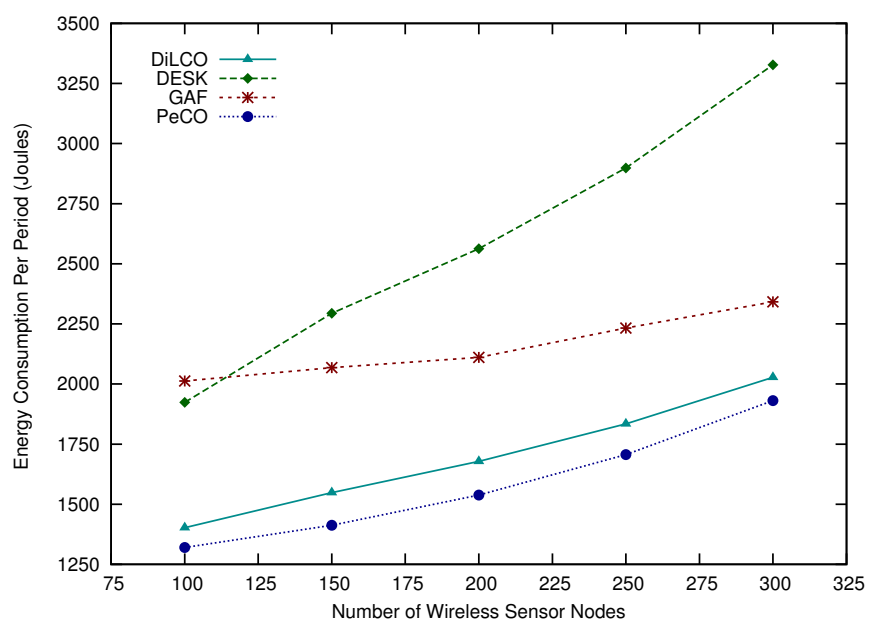

(a)

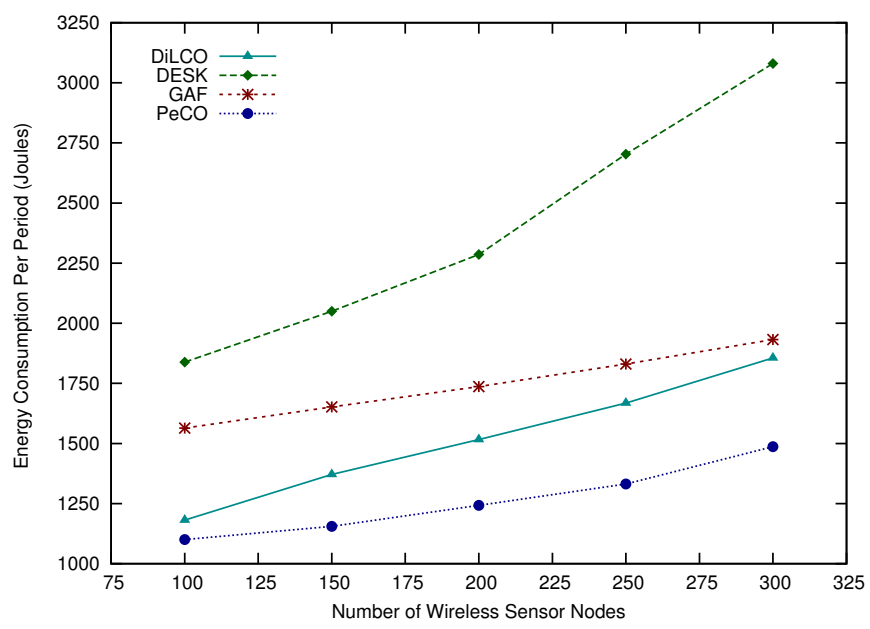

(b)

FIgURE 8 - Energy consumption per period for (a) Lifetime 95 and (b) Lifetime 50 .

Lifetime $_{95}$ and Lifetime $_{50}$ are shown for different network sizes. As can be seen in these figures, the lifetime increases with the size of the network, and it is clearly larger for the DiLCO and PeCO protocols. For instance, for a network of 300 sensors and coverage ratio greater than $50 \%$, it can be observed on Figure 9 (b) that the lifetime is about twice longer with $\mathrm{PeCO}$ compared to the DESK protocol. The performance difference is more obvious in Figure 9(b) than in Figure 9(a) because the gain induced by protocols (PeCO and DiLCO) increases with time, and the lifetime with a coverage over $50 \%$ is far longer than with $95 \%$.

Figure 10 compares the lifetime coverage of the DiLCO and PeCO protocols for different coverage ratios. Protocol/70, Protocol/80, Protocol/85, Pro- 


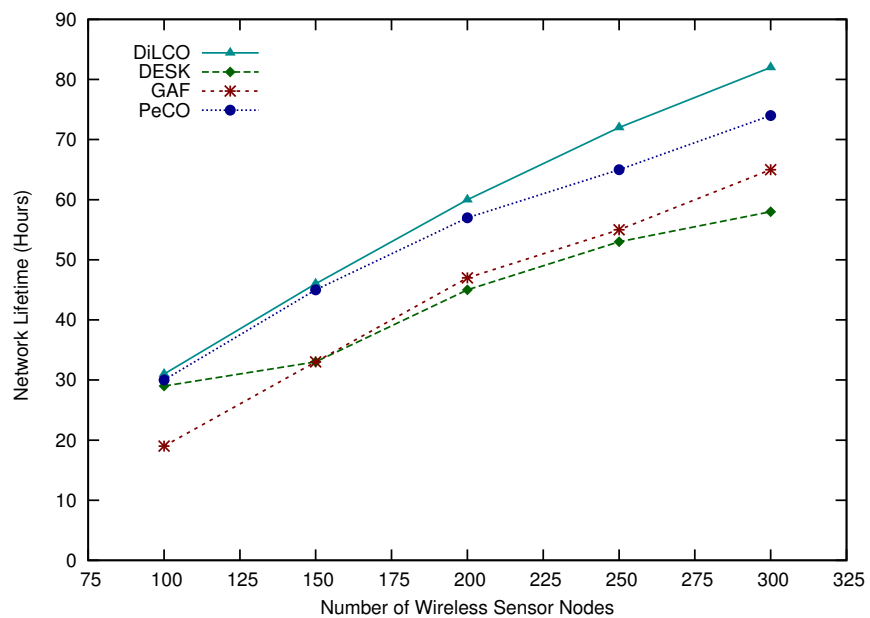

(a)

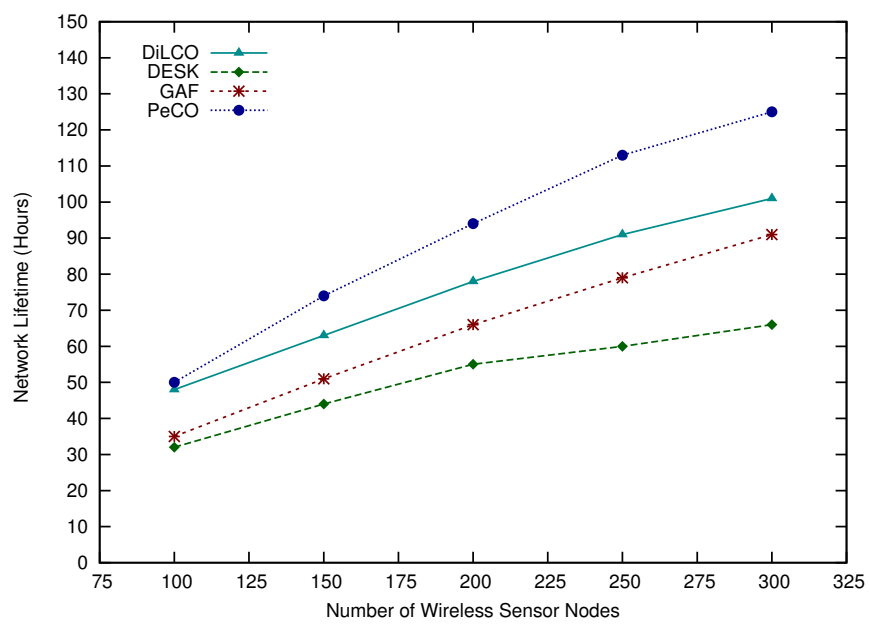

(b)

Figure 9 - Network Lifetime for (a) Lifetime $_{95}$ and (b) Lifetime 50.

tocol $/ 90$, and Protocol/95 correspond to the amount of time during which the network can satisfy an area coverage greater than $70 \%, 80 \%, 85 \%, 90 \%$, and 95\% respectively, where the term Protocol refers to DiLCO or PeCO. Indeed there are applications that do not require a $100 \%$ coverage of the area to be monitored. For example, forest fire application might require complete coverage in summer seasons while only require $80 \%$ of the area to be covered in rainy seasons [21]. As another example, birds habit study requires only $70 \%$-coverage at nighttime when the birds are sleeping while requires 100\%-coverage at daytime when the birds are active [18]. PeCO always outperforms DiLCO for the three lower coverage ratios, moreover the improvements grow with the network size. DiLCO outperforms PeCO when the coverage ratio is required to be $>90 \%$, but $\mathrm{PeCO}$ extends the network lifetime significantly when coverage ratio can be 
relaxed.

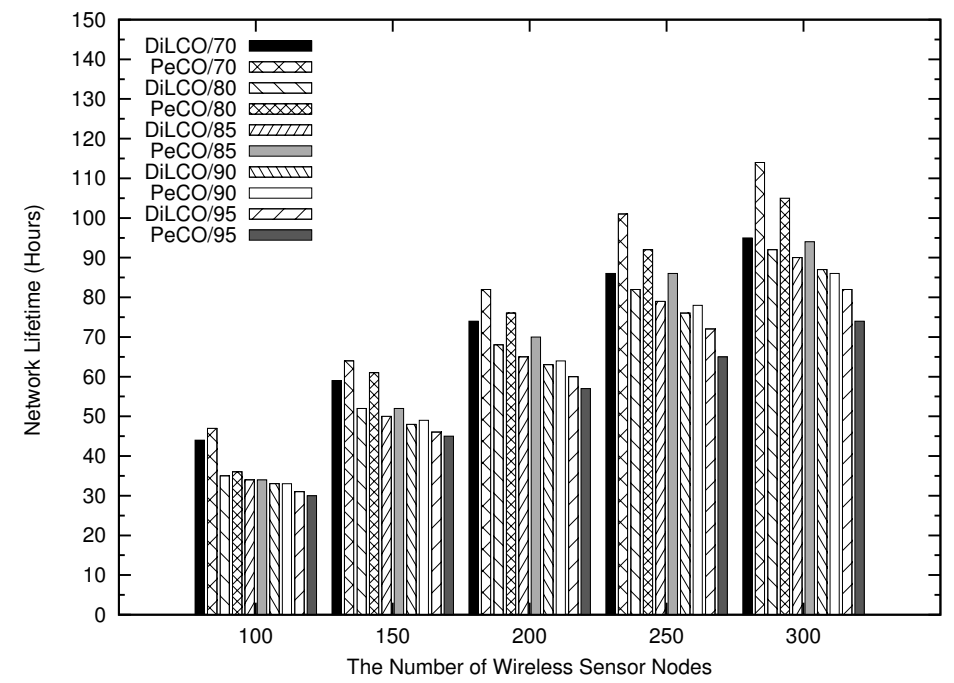

FiguRE 10 - Network lifetime for different coverage ratios.

\subsubsection{Impact of $\alpha$ and $\beta$ on PeCO's performance}

Table 4 shows network lifetime results for different values of $\alpha$ and $\beta$, and a network size equal to 200 sensor nodes. On the one hand, the choice of $\beta \gg \alpha$ prevents the overcoverage, and also limits the activation of a large number of sensors, but as $\alpha$ is low, some areas may be poorly covered. This explains the results obtained for Lifetime $_{50}$ with $\beta \gg \alpha$ : a large number of periods with low coverage ratio. On the other hand, when $\alpha \gg \beta$ is chosen, the coverage is favored even if some areas may be overcovered, so a high coverage ratio is reached, but a large number of sensors are activated to achieve this goal. Therefore the network lifetime is reduced. The choice $\alpha=0.6$ and $\beta=0.4$ seems to achieve the best compromise between lifetime and coverage ratio. That explains why this setting has been chosen for the experiments presented in the previous subsections.

\section{Conclusion and Future Works}

In this paper the problem of perimeter coverage optimization in WSNs has been studied. A new protocol called Perimeter-based Coverage Optimization is designed. This protocol schedules nodes' activities (wake up and sleep stages) with the objective of maintaining a good coverage ratio while maximizing the network lifetime. This protocol is applied in a distributed way in regular subregions obtained after partitioning the area of interest in a preliminary step. It works in periods and is based on the resolution of an integer program to select 
TABLE 4 - The impact of $\alpha$ and $\beta$ on PeCO's performance

\begin{tabular}{|c|c|c|c|}
\hline$\alpha$ & $\beta$ & Lifetime $_{50}$ & Lifetime $_{95}$ \\
\hline 0.0 & 1.0 & 151 & 0 \\
\hline 0.1 & 0.9 & 145 & 0 \\
\hline 0.2 & 0.8 & 140 & 0 \\
\hline 0.3 & 0.7 & 134 & 0 \\
\hline 0.4 & 0.6 & 125 & 0 \\
\hline 0.5 & 0.5 & 118 & 30 \\
\hline $\mathbf{0 . 6}$ & $\mathbf{0 . 4}$ & $\mathbf{9 4}$ & $\mathbf{5 7}$ \\
\hline 0.7 & 0.3 & 97 & 49 \\
\hline 0.8 & 0.2 & 90 & 52 \\
\hline 0.9 & 0.1 & 77 & 50 \\
\hline 1.0 & 0.0 & 60 & 44 \\
\hline
\end{tabular}

the subset of sensors operating in active status for each period. This work is original in so far as it proposes for the first time an integer program scheduling the activation of sensors based on their perimeter coverage level, instead of using a set of targets/points to be covered. Several simulations have been carried out to evaluate the proposed protocol. The simulation results show that $\mathrm{PeCO}$ is more energy-efficient than other approaches, with respect to lifetime, coverage ratio, active sensors ratio, and energy consumption.

This framework will be extented so that the schedules are planned for multiple sensing periods. The integer program would be improved to take into account heterogeneous sensors from both energy and node characteristics point of views. Finally, it would be interesting to implement the PeCO protocol using a sensor-testbed to evaluate it in real world applications.

\section{Acknowledgments}

Ali Kadhum Idrees' $\mathrm{PhD}$ thesis is financially supported in part by University of Babylon (Iraq). This work is also partially funded by the Labex ACTION program (contract ANR-11-LABX-01-01).

\section{Références}

[1] Ian F Akyildiz, Weilian Su, Yogesh Sankarasubramaniam, and Erdal Cayirci. Wireless sensor networks : a survey. Computer networks, 38(4) :393-422, 2002.

[2] Giuseppe Anastasi, Marco Conti, Mario Di Francesco, and Andrea Passarella. Energy conservation in wireless sensor networks : A survey. Ad Hoc Networks, 7(3) :537-568, 2009. 
[3] P. Berman and G. Calinescu. Power efficient monitoring management in sensor networks. In Proceedings of IEEE Wireless Communication and Networking Conference (WCNC'04), pages 2329-2334, 2004.

[4] Mihaela Cardei and Ding-Zhu Du. Improving wireless sensor network lifetime through power aware organization. Wireless Networks, 11(3) :333-340, 2005.

[5] Mihaela Cardei, My T Thai, Yingshu Li, and Weili Wu. Energy-efficient target coverage in wireless sensor networks. In INFOCOM 2005. 24th Annual Joint Conference of the IEEE Computer and Communications Societies. Proceedings IEEE, volume 3, pages 1976-1984, 2005.

[6] Fabian Castaño, André Rossi, Marc Sevaux, and Nubia Velasco. A column generation approach to extend lifetime in wireless sensor networks with coverage and connectivity constraints. Computers $\&$ Operations Research, 52(B) :220-230, 2014.

[7] Optimizer CPLEX. Ibm ilog cplex optimizer. Available : http ://www01.ibm.com/software/integration/optimization/cplex-optimizer/, 2010.

[8] Xiu Deng, Dongxiao Yu Jiguo Yu, and Congcong Chen. Transforming area coverage to target coverage to maintain coverage and connectivity for wireless sensor networks. International Journal of Distributed Sensor Networks, 2012, Article ID 254318 :1-12, 2012.

[9] Karine Deschinkel. A column generation based heuristic to extend lifetime in wireless sensor network. Sensors \& Transducers Journal, 14(2) :242-253, 2012.

[10] Robert Fourer, David M. Gay, and Brian W. Kernighan. AMPL : A Modeling Language for Mathematical Programming. Cengage Learning, 2nd edition, 2002.

[11] Shibo He, Xiaowen Gong, Junshan Zhang, Jiming Chen, and Youxian Sun. Curve-based deployment for barrier coverage in wireless sensor networks. IEEE Transactions on Wireless Communications, 13(2) :724-735, 2014.

[12] Chi-Fu Huang and Yu-Chee Tseng. The coverage problem in a wireless sensor network. Mobile Networks and Applications, 10(4) :519-528, 2005.

[13] Ka-Shun Hung and King-Shan Lui. Perimeter coverage scheduling in wireless sensor networks using sensors with a single continuous cover range. EURASIP Journal on Wireless Communications and Networking, 2010, 2010.

[14] Ali Kadhum Idrees, Karine Deschinkel, Michel Salomon, and Raphaël Couturier. Coverage and lifetime optimization in heterogeneous energy wireless sensor networks. In ICN 2014, The Thirteenth International Conference on Networks, pages 49-54, 2014.

[15] Ali Kadhum Idrees, Karine Deschinkel, Michel Salomon, and Raphaël Couturier. Distributed lifetime coverage optimization protocol in wireless sensor networks. Technical Report RR-FEMTO-ST-2623, University of FrancheComte - FEMTO-ST Institute, DISC Research Department, March 2015. 
[16] N. Jaggi and A.A. Abouzeid. Energy-efficient connected covereage in wireless sensor networks. In Proceeding of 4th Asian International Mobile Computing Conference AMOC2006, pages 77-86, 2006.

[17] Hyunbum Kim and Jorge A Cobb. Maximum lifetime of reinforced barriercoverage in wireless sensor networks. In 19th IEEE International Conference on Networks (ICON), 2013, pages 1-6, 2013.

[18] J. Kumagai. Life of birds [wireless sensor network for bird study]. Spectrum, IEEE, 41(4) :42-49, April 2004.

[19] Eva K Lee, Richard J Gallagher, David Silvern, Cheng-Shie Wuu, and Marco Zaider. Treatment planning for brachytherapy : an integer programming model, two computational approaches and experiments with permanent prostate implant planning. Physics in Medicine and Biology, 44(1) :145, 1999.

[20] Mo Li and Athanasios V Vasilakos. A survey on topology control in wireless sensor networks : Taxonomy, comparative study, and open issues. Proceedings of the IEEE, 101(12):2538-2557, 2013.

[21] Yingshu Li, Chinh Vu, Chunyu Ai, Guantao Chen, and Yi Zhao. Transforming complete coverage algorithms to partial coverage algorithms for wireless sensor networks. IEEE Transactions on Parallel and Distributed Systems, 22(4) :695-703, 2011.

[22] Hui Ling and Taieb Znati. Energy efficient adaptive sensing for dynamic coverage in wireless sensor networks. In Wireless Communications and Networking Conference, 2009. WCNC 2009. IEEE, pages 1-6, 2009.

[23] Andrew Makhorin. The glpk (gnu linear programming kit). Available : https ://www.gnu.org/software/glpk/, 2012.

[24] S. Misra, M. P. Kumar, and M. S. Obaidat. Connectivity preserving localized coverage algorithm for area monitoring using wireless sensor networks. Computer Communications, 34(12) :1484-1496, 2011.

[25] T.V. Padmavathy and M. Chitra. Extending the network lifetime of wireless sensor networks using residual energy extraction-hybrid scheduling algorithm. Int. J. of Communications, Network and System Sciences, 3(1) :98106, 2010.

[26] Daniele Puccinelli and Martin Haenggi. Wireless sensor networks : applications and challenges of ubiquitous sensing. IEEE Circuits and Systems Magazine, 5(3) :19-31, 2005.

[27] Arun K Pujari. High-energy-first (hef) heuristic for energy-efficient target coverage problem. International Journal of Ad Hoc, Sensor $\&$ Ubiquitous Computing, 2(1) :45-58, 2011.

[28] Yipeng Qu and Stavros V Georgakopoulos. A distributed area coverage algorithm for maintenance of randomly distributed sensors with adjustable sensing range. In IEEE Global Communications Conference (GLOBECOM), 2013, pages 286-291, 2013. 
[29] Tifenn Rault, Abdelmadjid Bouabdallah, and Yacine Challal. Energy efficiency in wireless sensor networks : A top-down survey. Computer Networks, $67: 104-122,2014$.

[30] Alok Singh, AndrÃl Rossi, and Marc Sevaux. Metaheuristic approaches for q-coverage problem versions in wireless sensor networks. Engineering Optimization, 45(5) :609-626, 2013.

[31] A. Varga. Omnet ++ discrete event simulation system. Available : http ://www.omnetpp.org, 2003.

[32] C. T. Vu. Distributed energy-efficient solutions for area coverage problems in wireless sensor networks. PhD thesis, Georgia State University, 2009.

[33] Chinh Vu, Shan Gao, Wiwek Deshmukh, and Yingshu Li. Distributed energy-efficient scheduling approach for k-coverage in wireless sensor networks. In IEEE Military Communications Conference, 2006. MILCOM 2006, pages 1-7, 2006.

[34] Bang Wang. Coverage problems in sensor networks : A survey. ACM Computing Surveys (CSUR), 43(4) :32-53, 2011.

[35] Xiaofei Xing, Jie Li, and Guojun Wang. Integer programming scheme for target coverage in heterogeneous wireless sensor networks. In Mobile Adhoc and Sensor Networks (MSN), 2010 Sixth International Conference on, pages 79-84, 2010.

[36] Ya Xu, John Heidemann, and Deborah Estrin. Geography-informed energy conservation for ad hoc routing. In Proceedings of the 7th annual international conference on Mobile computing and networking, pages 70-84, 2001.

[37] Ting Yan, Yu Gu, Tian He, and John A Stankovic. Design and optimization of distributed sensing coverage in wireless sensor networks. ACM Transactions on Embedded Computing Systems (TECS), 7(3):33, 2008.

[38] Changlin Yang and Kwan-Wu Chin. Novel algorithms for complete targets coverage in energy harvesting wireless sensor networks. IEEE Communications Letters, 18(1) :118-121, 2014.

[39] Changlin Yang and Kwan-Wu Chin. A novel distributed algorithm for complete targets coverage in energy harvesting wireless sensor networks. In IEEE ICC 2014- Ad-hoc and Sensor Networking Symposium, pages 361366, 2014.

[40] Mengmeng Yang and Jie Liu. A maximum lifetime coverage algorithm based on linear programming. Journal of Information Hiding an dMultimedia Signal Processing, Ubiquitous International, 5(2) :296-301, 2014.

[41] Jennifer Yick, Biswanath Mukherjee, and Dipak Ghosal. Wireless sensor network survey. Computer networks, 52(12) :2292-2330, 2008.

[42] H. Zhang and J. C. Hou. Maintaining sensing coverage and connectivity in large sensor networks. Ad Hoc $B 3$ Sensor Wireless Networks, 1(1-2), 2005.

[43] Zongheng Zhou, Samir R Das, and Himanshu Gupta. Variable radii connected sensor cover in sensor networks. ACM Transactions on Sensor Networks (TOSN), 5(1) :8, 2009. 
[44] Dimitrios Zorbas, Dimitris Glynos, Panayiotis Kotzanikolaou, and Christos Douligeris. Solving coverage problems in wireless sensor networks using cover sets. Ad Hoc Networks, 8(4) :400-415, 2010. 